\title{
EUROfusion
}

EUROFUSION WPJET1-PR(16)14851

M. Schneider et al.

\section{Modelling Third Harmonic Ion Cyclotron Acceleration of Deuterium Beams for JET Fusion Product Studies Experiments}


"This document is intended for publication in the open literature. It is made available on the clear understanding that it may not be further circulated and extracts or references may not be published prior to publication of the original when applicable, or without the consent of the Publications Officer, EUROfusion Programme Management Unit, Culham Science Centre, Abingdon, Oxon, OX14 3DB, UK or e-mail Publications.Officer@euro-fusion.org".

"Enquiries about Copyright and reproduction should be addressed to the Publications Officer, EUROfusion Programme Management Unit, Culham Science Centre, Abingdon, Oxon, OX14 3DB, UK or e-mail Publications.Officer@euro-fusion.org".

The contents of this preprint and all other EUROfusion Preprints, Reports and Conference Papers are available to view online free at http://www.euro-fusionscipub.org. This site has full search facilities and e-mail alert options. In the JET specific papers the diagrams contained within the PDFs on this site are hyperlinked. 


\title{
Modelling third harmonic Ion Cyclotron acceleration of deuterium beams for JET Fusion Product Studies Experiments
}

\author{
M. Schneider ${ }^{1}$, T. Johnson ${ }^{2}$, R. Dumont ${ }^{1}$ J. Eriksson ${ }^{3}$, L.-G. Eriksson ${ }^{4}$, L. Giacomelli ${ }^{5}$, J.-B. \\ Girardo $^{1}$, T. Hellsten ${ }^{2}$, E. Khilkevitch ${ }^{6}$, V.G. Kiptily ${ }^{5}$, T. Koskela ${ }^{7}$, M. Mantsinen $^{8,9}$, M. \\ Nocente $^{10}$, M. Salewski ${ }^{11}$, S.E. Sharapov ${ }^{5}$, A.E. Shevelev ${ }^{6}$, and JET contributors* \\ EUROfusion Consortium, JET, Culham Science Centre Abingdon, OX14 3DB, UK \\ ${ }^{1}$ CEA, IRFM, F-13108 Saint-Paul-lez-Durance, France \\ ${ }^{2}$ KTH Royal Institute of Technology, 10044 Stockholm, Sweden \\ ${ }^{3}$ Dept. of Physics and Astronomy, Uppsala University, Sweden \\ ${ }^{4}$ Europ. Commission, Directorate General for Research and Innov., B-1049 Brussels, Belgium \\ ${ }^{5}$ CCFE, JET, Culham Science Centre, Abingdon, OX14 3DB, UK \\ ${ }^{6}$ Ioffe Institute, St. Petersburg, 194021 Russia \\ ${ }^{7}$ Department of Applied Physics, Aalto University, FI- 00076 AALTO, Finland \\ ${ }^{8}$ Catalan Institution for Research and Advanced Studies, Barcelona, Spain \\ ${ }^{9}$ Barcelona Supercomp. Center, Barcelona, Spain \\ ${ }^{10}$ Dipartimento di Fisica "G. Occhialini”, Università degli Studi di Milano-Bicocca, Milano, Italy \\ 11 Technical University of Denmark, Department of Physics, DK-2800 Kgs. Lyngby, Denmark
}

\begin{abstract}
Recent JET experiments have been dedicated to the studies of fusion reactions between Deuterium $(D)$ and Helium-3 $\left({ }^{3} \mathrm{He}\right)$ ions using Neutral Beam Injection (NBI) in synergy with third harmonic Ion Cyclotron Radio-frequency Heating (ICRH) of the beam. This scenario generates a fast ion deuterium tail enhancing $D(D, n)^{3} H e$ and $D\left({ }^{3} H e, p\right) \alpha$ fusion reactions (referred to as $D D$ and $D^{3} \mathrm{He}$ reactions for the rest of the paper). Modelling and measuring the fast deuterium tail accurately is essential for quantifying the fusion products. This paper presents the modelling of the $D$ distribution function resulting from the NBI + ICRF heating scheme, reinforced by a comparison with dedicated JET fast ion diagnostics, showing an overall good agreement. Finally, a sawtooth activity for these experiments has been observed and interpreted using SPOT/RFOF simulations in the framework of Porcelli's theoretical model, where NBI+ICRH accelerated ions are found to have a strong stabilizing effect, leading to monster sawteeth.
\end{abstract}

\section{Introduction}

Fusion products resulting from $T(D, n)^{4} \alpha$ reactions will play a crucial role in future tokamak fusion devices. In particular, the plasma heating in a reactor must be sustained by the power transfer from fusion born alpha particles to the thermal plasma through collisional slowing down. Other fusion products can be used for diagnostic purposes. For this reason it is essential to develop a comprehensive understanding of the physics governing their behaviour. JET experiments have a long history of being at the forefront of this quest, with the DT campaign in 1997 [1] producing important results on plasma heating by fusion-born alpha particles $[2,3]$. Alpha particles were however better diagnosed in TFTR, providing essential results on alpha physics $[4,5]$. There have also been other studies of energetic $H e$ ions in JET, notable examples are those in Ref. [6, 7],

* See the Appendix of F. Romanelli et al., Proceedings of the 25th IAEA Fusion Energy Conference 2014, Saint Petersburg, Russia. 
where third harmonic ICRF heating was used to accelerate neutral beam injected $H e$ ions to $\mathrm{MeV}$ energies.

The 2014 JET fusion product studies (FPS) experiments were aimed at studying the confinement of nuclear products and exploring the detection capabilities of JET neutron and fast ion diagnostics, with a view to guide the development of ITER neutron diagnostics and diagnostics for detecting confined and lost fast ions. Consequently, JET fusion products in the experiments should, as much as possible, have similar characteristics to ITER fusion-born alpha particles originating in $D T$ reactions. In JET deuterium plasmas, the best candidate is to introduce some ${ }^{3} \mathrm{He}$, which leads to the production of $3.7 \mathrm{MeV}$ alpha particles from $D\left({ }^{3} \mathrm{He}, p\right) \alpha$ reactions $\left(D^{3} \mathrm{He}\right)$, i.e. very similar to $3.5 \mathrm{MeV}$ alpha particles from $D T$ reactions. However, the fusion cross section for this reaction peaks at much higher center-of-mass-energy than for $D T$ reactions as shown in Fig.(1) extracted from [8], i.e. MeV-range ions are necessary to enhance fusion reactivity to make the reaction useful for FPS. Only ICRH can efficiently produce such energetic ions in the JET tokamak. Fundamental $D$ heating is not an option since the amplitude of the left-hand polarized Radio-Frequency (RF) electric field has a minimum around the cold ion-cyclotron resonance layer of the majority ions due to screening. Second harmonic $D$ heating is possible but there is always some residual hydrogen in the plasma which tends to absorb a large fraction of the ICRF power at the fundamental $H$ resonance layer, which coincides with the $\omega=2 \omega_{c D}$ layer, where $\omega_{c D}$ is the cyclotron frequency of deuterium, see e.g. JET second harmonic $D$ heating experiments reported in $[9]$.

While ${ }^{3} \mathrm{He}$ minority heating is a viable option [10], it has the drawback that the ${ }^{3} \mathrm{He}$ concentration must be kept low, around a few percent, for efficient ${ }^{3} \mathrm{He}$ absorption. Hence, the best option is to use third harmonic ICRF heating for creating energetic $D$ ions, which is similar to the acceleration of alpha particles reported in [6], where neutral beam injection of the resonating species was used to improve wave absorption process. NBI is indeed highly beneficial as the efficiency of the third harmonic ICRF heating scheme depends on the Larmor radius of the resonating species as will be discussed in section 3. It should be noted that for the discharges analysed in the present paper ${ }^{3} \mathrm{He}$ was not introduced, i.e. only the efficiency of the third harmonic $D$ scenario was explored. In other discharges of the campaign ${ }^{3} \mathrm{He}$ was introduced; an analysis of the ICRF physics associated with them can be found in Ref. [11].

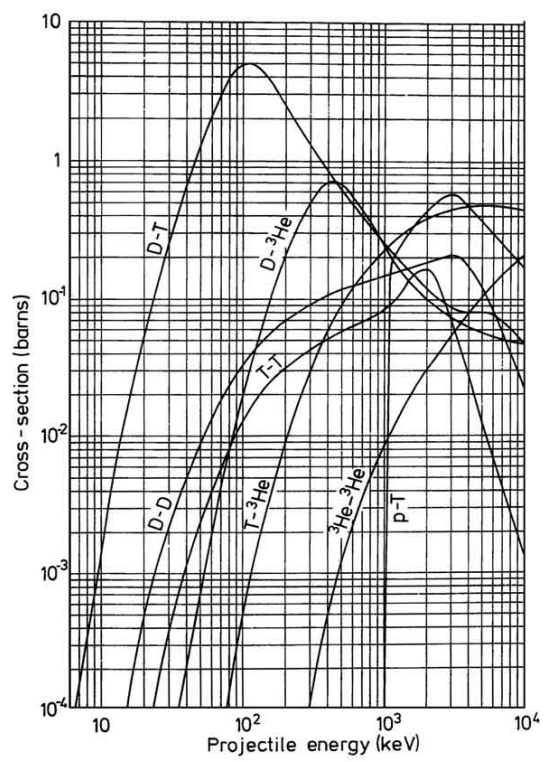

Figure 1: Fusion reaction cross-sections (figure extracted from [8]).

In addition to serve as discharges for studying energetic particle physics, the experiments with third harmonic ICRF heating are also valuable for the process of validating modelling of fast particle distribution functions and their influence on the ICRF wave propagation. It is this latter aspect that is the focus of the present paper. Here both neutron diagnostics [12] and gamma-ray diagnostics [13, 14] play a crucial role in providing data that the modelling can be compared with.

Modelling of fast ions accelerated by ICRF waves is a highly complex task. It requires solving 
Maxwell's equations for the wave field in the plasma and simulating the distribution functions of all species absorbing wave power. Thus formally one should solve:

$$
\begin{gathered}
\nabla \times \nabla \times \vec{E}=-\frac{\omega^{2}}{c^{2}} \stackrel{\leftrightarrow}{\epsilon}\left(f_{i}, \ldots, f_{n_{\text {res }}}\right) \cdot \vec{E}-i \omega \mu_{0} j_{\text {ext }} \\
\frac{d f_{i}}{d t}=C\left(f_{i}\right)+Q\left(f_{i}\right)+S_{N B I}, \text { for } i=1, \ldots, n_{\text {res }},
\end{gathered}
$$

self-consistently. Here $\stackrel{\leftrightarrow}{\epsilon}$ is the dielectric tensor that depends on the $n_{\text {res }}$ distributions functions, $f_{i}$, of the resonating species - which may deviate significantly from Maxwellian - and of course all other plasma species; $\omega$ is the wave frequency, $c$ is the speed of light, $\vec{E}$ is the wave electric field, $\mu_{0}$ is the vacuum magnetic permeability and $j_{e x t}$ is the current density in the antenna; $C\left(f_{i}\right)$ is a Fokker-Planck collision operator, $Q\left(f_{i}\right)$ is an operator describing the wave-particle interaction and $S_{N B I}$ represents a source of neutral beam injected ions. The distribution function is typically gyro averaged or further reduced in dimension by orbit averaging (in the former case $d / d t=$ $\partial / \partial t+\vec{v}_{D} \cdot \nabla f_{i}$, where $\vec{v}_{D}$ is the ion drift velocity, while in the latter we simply have $d / d t=\partial / \partial t$ ). To solve these two equations self-consistently is a rather formidable problem, especially since both equations require highly sophisticated codes to obtain results with a high degree of physics fidelity. For this reason very few simulation codes have attempted to resolve this combined problem selfconsistently. At JET the perhaps most utilised codes capable of self-consistent ICRF calculations are PION [9] and SELFO [15]. However, both these codes have shortcomings. The PION code is based on significantly simplified models both for the wave propagation and the Fokker-Planck modelling of the distribution functions (despite this it is has been quite successful in modelling ICRF heating on JET, see e.g. [16]). The SELFO code, on the other hand, assumes a simplified geometry in its Fokker-Planck treatment of the resonating ion species. Consequently, there is significant scope for improving the modelling of ICRF heating, and it is a highly active area within the EUROfusion Code Development for Integrated Modelling project (WPCD). In particular, this project aims at integrating a full set of fusion plasma simulation codes within the EU Integrated Modelling (EU-IM) framework [17], a platform in which codes can be integrated in a standardised form.

While there are several full-wave codes available within the EU-IM framework [18, 19, 20, 21], there is a more limited choice of codes capable of solving the Fokker-Planck equation including ICRF interaction, full orbit effects and general geometry. In fact there were none in the EU-IM framework until work began on developing a new library called RFOF [22], which is an operator that can be called from orbit following Monte Carlo codes to update the Monte Carlo markers' (test particles) positions in phase space due to ICRF wave-particle interaction. The SPOT [23] and ASCOT [24] orbit following Monte Carlo codes, available within the EU-IM framwork, have recently been augmented with the RFOF library. Both the SPOT/RFOF and ASCOT/RFOF packages can be run in combination with any wave code in the EU-IM framework. SPOT/RFOF has recently been run together with the EVE full wave code for ICRF second harmonic tritium heating in the context of an ITER ICRF scenario modelling [25]. However, the option of incorporating dielectric properties derived from Monte Carlo markers into the full wave codes of the EU-IM framework, similar to SELFO, is as yet not routinely available (work in this direction has been initiated [26]). Before embarking on deeper integration with wave deposition codes, the current priorities for ICRF modelling within the WPCD project, are (i) further development of RFOF and interfacing to SPOT and ASCOT; (ii) verification and (limited) validation of the combined SPOT/RFOF 
and ASCOT/RFOF packages. It is this latter activity that is the focus of the present paper. For the analysis presented here, SPOT/RFOF (ASCOT/RFOF) uses wave characteristics derived from simulations by the PION code as an input [27], along with the NEMO [28] (BBNBI [29]) beam deposition code to simulate relevant JET discharges including combined NBI and ICRF. As discussed above, the experimental scenario analysed is neutral beam injection combined with ICRF waves tuned to the third harmonic deuterium cyclotron frequency in the plasma centre. This is technically a quite difficult scenario to simulate, and serves as a stringent test for benchmarking the combination of RFOF and the two Monte Carlo codes against each other. Furthermore, with detailed fast particle diagnostics available at JET, it is possible to carry out limited validation (limited because output from the PION code is used instead of a true self-consistent calculation with the output of SPOT or ASCOT fed back to a wave deposition code, and also because validation must be an ongoing process, which has just started for SPOT/RFOF and ASCOT/RFOF). The validation effort in this paper is concentrated on a comparison between the deuterium distribution function simulated with the SPOT/RFOF and ASCOT/RFOF modelling packages and the neutron

and gamma-ray diagnostics via a dedicated synthetic description. Furthermore, the deuterium energy distribution is compared between different modelling tools and de-convoluted data from neutron and gamma-ray spectroscopy. To demonstrate further integration and validation work, the output of SPOT/RFOF has also been used to investigate sawtooth stabilisation due to the ICRF accelerated fast ions.

This paper is organised as follows: the second section presents the experimental conditions implying a scenario with synergy between NBI and ICRH third harmonic deuterium heating. The third section describes the absorption mechanism of this heating scheme. The fourth section presents the modelling tools and the method for combining them in the context of this analysis. The fifth section shows the features and results of the simulations. The sixth section presents the comparison between modelling and neutron and gamma synthetic diagnostics, while the seventh section shows the comparison of modelled and experimental deuterium energy distributions. The eighth section briefly describes the sawtooth analysis carried out around these experiments using the same modelling tools. Finally, results are discussed and conclusions are drawn in the ninth section.

\section{Experimental scenario: $D$ NBI and $\omega=3 \omega_{c D}$ ICRF heat- ing}

For the experiment analysed here the central magnetic field was $2.24 \mathrm{~T}$ and a frequency of 51 $\mathrm{MHz}$ was chosen for the ICRF waves, positioning the third harmonic $D$ resonance near the plasma centre, which is in the line of sight of most of the neutron diagnostics. At the very edge, a parasitic absorption may occur on the high field side, where the resonance layer for the fundamental $(n=1)$ interaction with $H$ ions is located (superimposed with the $D$ second harmonic heating, $n=2$ ). The main competition for the ICRF power should be between the third harmonic $D$ absorption and direct electron damping via Transit Time Magnetic Pumping and Electron Landau Damping (TTMP/ELD). However, in order to obtain consistency between measured and simulated DD neutron rates, a parasitic absorption mechanism had to be introduced in the PION simulations [27], which indicates that the hydrogen resonance near the edge and/or some other parasitic effect also played a role.

The auxiliary heating scheme and the $D D$ neutron rate are displayed in Figure 2 along with ion 

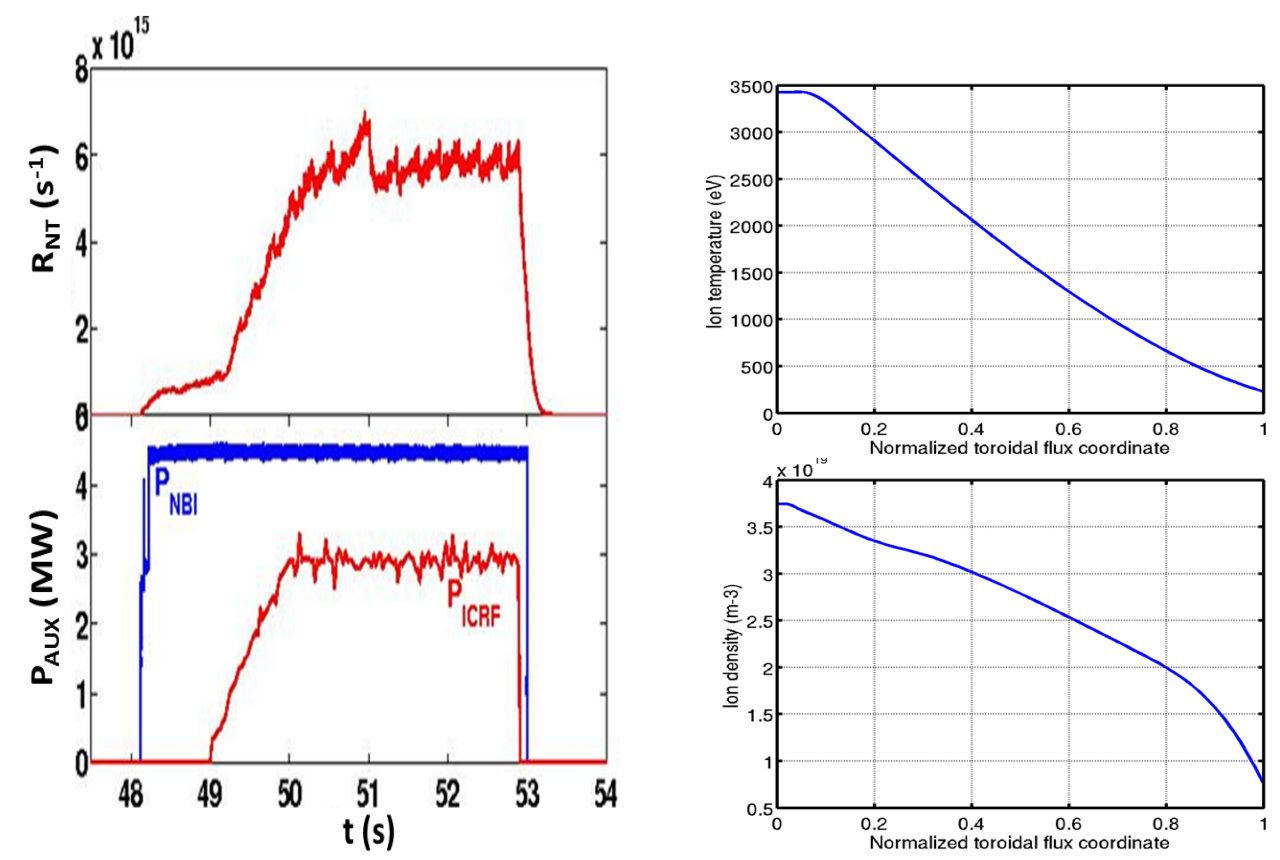

Figure 2: Time evolution of neutron rate $R_{N T}$, with a $D D$ fusion rate $R_{D D}=2 R_{N T}$ (top-left figure), applied NBI and ICRH power (bottom-left figure), ion temperature profile (top-right figure) and ion density profile (bottom-right figure) for discharge \#86459.

temperature and density profiles, for JET discharge \#86459, which was typical of the discharges analysed in FPS experiments. The effect on the neutron rate by the introduction of only $3 \mathrm{MW}$ of ICRF power is quite spectacular. It increases by about a factor of six compared to the NBI only phase. From this we can conclude that the ICRF power must have been quite effective in accelerating deuterons to the $\mathrm{MeV}$ range. In the next section we discuss qualitatively the main factors behind mechanism for this acceleration.

\section{$3 \quad$ Absorption mechanism at $\omega=3 \omega_{c D}$ ICRF heating}

The electric field component accelerating the resonating ions is to lowest order the left hand polarised component, $E_{+}$, which rotates in the ion Larmor motion direction. When the wave frequency is a harmonic of the ion cyclotron frequency $\left(\omega=n \omega_{c i}, n \geq 2\right)$, the $E_{+}$component completes $n$ full revolutions during one Larmor orbit of a resonating ion. As a result, during the completion of a Larmor orbit, an ion will experience a sequence of accelerations and de-accelerations (there will in fact be $n-1$ pairs of arcs along the orbit with acceleration followed by de-acceleration). If the $E_{+}$component of the electric field does not vary along the Larmor orbit the acceleration will be exactly cancelled by de-acceleration and the perpendicular velocity of the resonating ions will effectively be unaffected. However, since the ICRF wave propagates perpendicular to the magnetic field there is a variation of $E_{+}$along the Larmor orbit, the strength of which is characterised by $k_{\perp} \rho$, where $k_{\perp}$ is the perpendicular wave number and $\rho=v_{\perp} / \omega_{c i}$ is the ion Larmor radius. Thus, owing to Finite Larmor Radius (FLR) effects there will not be complete cancellation, instead an ion will receive a finite "kick" in its perpendicular velocity $v_{\perp}$, as a result of the wave-particle interaction.

Because of the variation of the magnetic field with the major radius position in a tokamak, 


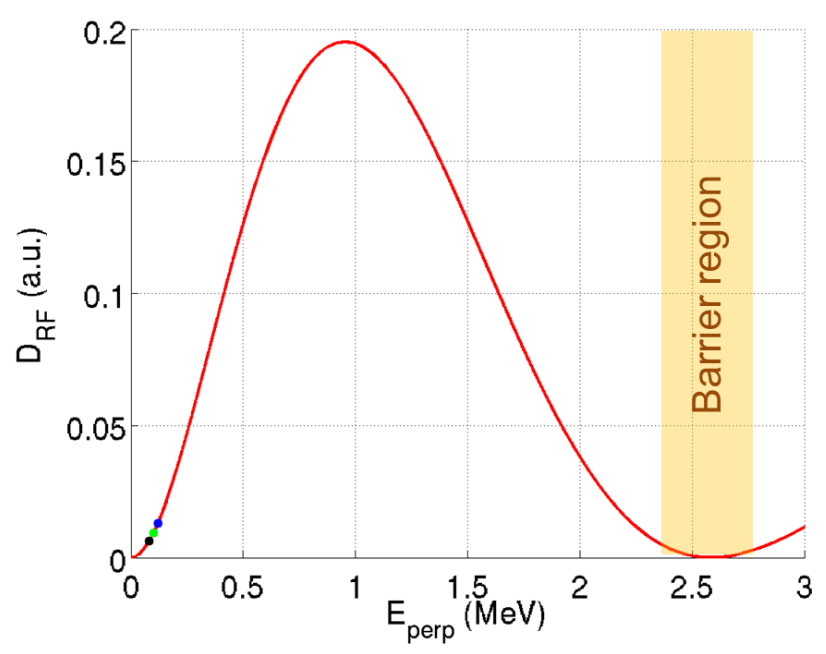

Figure 3: Energy dependence of the diffusion coefficient $D_{R F}$ via Bessel functions for the third harmonic heating, using the wave conditions of the present JET experiments. The three points at low perpendicular energy represents the beam injection energies, for which the RF diffusion coefficient is low.

a resonating ion will be in actual resonance with the wave only in a few places along its guiding centre orbit where the Doppler shifted wave frequency matches the harmonic of the ion cyclotron frequency, i.e. in the vicinity of where $\omega-k_{\|} v_{\|}=n \omega_{c i}$, defining a vertical resonance layer, where $k_{\|}$and $v_{\|}$are the parallel wave vector and velocity respectively. For resonating passing ions there are two resonances along an orbit for a given toroidal mode number whereas there are two to four for resonating trapped ions. As a result, a resonating ion will receive a finite number of discrete "kicks" in its perpendicular velocity $\Delta v_{\perp}$ during a poloidal revolution of its guiding centre orbit. If these kicks are uncorrelated, which they tend to be since collisions and non-linear effects usually are sufficient to scramble the phase memory of the ions with respect to the wave phase between resonance interactions, there will be a random walk process in velocity space which can be approximately characterised by an ICRF induced diffusion term normalized to the bounce frequency, $D_{R F}\left\langle\Delta v_{\perp}^{2}\right\rangle / \tau_{b}$, where $\tau_{b}$ is the bounce time, i.e. the time to complete a poloidal revolution of a guiding centre orbit [30].

A careful analysis of the FLR dependence of $D_{R F}$ shows:

$$
D_{R F} \propto\left|E_{+} J_{n-1}\left(\frac{k_{\perp} v_{\perp}}{\omega_{c i}}\right)+E_{-} J_{n+1}\left(\frac{k_{\perp} v_{\perp}}{\omega_{c i}}\right)\right|^{2},
$$

where $E_{+}$and $E_{-}$are the left and right-handed components of the wave electric field (estimated by the wave code), and $J_{n}$ are Bessel functions of the first kind. The $D_{R F}$ dependence of Eq.(3) is displayed in Fig.(3) for $n=3$. As can be seen, the wave-particle interaction strength is weak for low energy particles, and since $D_{R F}$ is also intimately linked to the power absorption, one can understand that the wave damping on thermal ions for third harmonic heating is relatively weak (this is at least true for JET conditions). A strong wave-particle interaction requires relatively energetic ions, i.e. the third harmonic absorption will be more efficient if NBI is used to create energetic ions that can set up a "seed absorptivity". This is the reason why the experiments reported here used combined NBI and ICRF.

However, the presence of NBI is not strictly necessary for third harmonic heating to be efficient, 
as demonstrated in [10]. Instead it requires a higher ICRH power than was available for the present experiments. In the experiments described in [10], the ICRH power was high enough to establish a fast ion tail despite the low absorption on the thermal ions. This tail was self-enhanced by the increasing absorption by the higher energy $D$ ions, such that most of the ICRF power was absorbed via third harmonic heating in the end. The same self-enhanced absorption phenomena is present also during combined NBI and third harmonic ICRF heating, and one therefore expects the resonating ions to accelerate to very high energies.

Coming back to the FLR dependence of $D_{R F}$, as demonstrated in Fig.(3), FLR effects will eventually start to reduce the wave-particle interaction strength after having reached a maximum, and at some point $D_{R F}$ becomes very small (in reality it will never go exactly to zero because the ICRF antennas emit a spectrum of toroidal modes, which all have slightly different $k_{\perp}$ ) effectively introducing a barrier in the energy of the fast ions, beyond which very few resonating ions will be accelerated by the ICRF waves (this effect has been demonstrated on JET before, see [31, 32]). Thus, the self-enhanced absorption process saturates once high energy ions have reached the barrier region. Furthermore, the barrier region ensures that there is no significant overshoot of the D tail ions beyond the peak of the $D D$ fusion cross section as can be seen in Fig.(3) for parameters representative of the discharges analysed in the present paper. Thus, the combined scheme of neutral beam injection of $D$ ions and $\omega=3 \omega_{c D}$ ICRF heating is very effective at producing $D D$ fusion reactions, which explains the spectacular rise in the $D D$ neutron rate with ICRF power shown in Fig.(2). An advantage of this substantial fast ion generated neutron rate is that the fast ion distribution function can be well diagnosed by neutron diagnostics. Moreover, this heating scheme serves as stringent scenario for validation of ICRF modelling codes. In particular, it does not leave any real "free parameters" in the simulations (in contrast to cases with minority heating, where the profile of the minority species can seldom be obtained with good accuracy), at the same time it requires that many quantities are correctly calculated, e.g. those that are key in determining the location in phase space of the "barrier region" discussed above.

\section{Fokker-Planck Modelling for NBI and ICRF heating}

The NBI ions source term in the Fokker-Planck equation (2) is modelled using the NEMO and BBNBI beam deposition codes. The former is based on a narrow beam model while the latter is based on the Monte Carlo technique; they have been shown to agree well [33]. The Fokker-Planck equation is solved using either SPOT or ASCOT orbit following Monte Carlo codes. They follow guiding centre orbits and use a Monte Carlo operator to account for collisions between the marker particles and the bulk plasma species. SPOT and ASCOT have recently been augmented with the RFOF library to model the interaction between ions and ICRF waves. Using two independent Fokker-Planck codes helps the interpretation of the prospective differences between modelling and experiment, allowing the identification of systematic errors due e.g. to kinetic profile measurements, or specific errors due to the model itself or the implementation of the RFOF quasilinear operator.

In order to speed up the simulations with orbit following Monte Carlo codes, so called accelerated collisions are often employed. This is possible if the particle orbits are only weakly perturbed after one poloidal revolution, i.e. after a bounce time $\tau_{b}$. In this case one simulated

poloidal revolution of an orbit can be used to represent $N_{a c c}$ real ones by applying a Monte Carlo collision operator that represents the accumulated effect of collisions during $N_{\text {acc }}$ passages of an orbit segment (the collision Monte Carlo operator is normally applied after each time step along 
the orbit). This means that phase information along the orbit is effectively lost and the resulting solution will be equivalent to solving an orbit averaged Fokker-Planck equation. This latter fact is employed to develop an operator for wave-particle interaction when accelerated collisions are used. From the theory of the orbit averaged quasi-linear operators one can construct an operator for each individual resonance point (where $\omega-n \omega_{c i}-k_{\|} v_{\|}-k_{\perp} V_{D}=0$ ) from which the kick in the phase space variables can be derived for $N_{a c c}$ passages of a resonance point. In an orbit averaged quasi-linear operator a particle orbit is characterised by three invariants of the unperturbed orbit, and we adopt the following:

$$
\left\{\begin{aligned}
I_{\perp} & =\frac{\omega B}{n \omega_{c i}} \mu \\
I_{\|} & =W-I_{\perp} \\
I_{\phi} & =P_{\phi}-\frac{N}{\omega} W
\end{aligned}\right.
$$

where $B$ is the local magnetic field, $\mu$ is the magnetic moment, $W$ is the ion kinetic energy, $P_{\phi}$ is the canonical toroidal angular momentum and $N$ is the wave toroidal mode number. In these invariants one can show that for a given toroidal mode number the wave-particle interaction only takes place along the $I_{\perp}$ direction in phase space, i.e. passage of a resonance point will give rise to a $\Delta I_{\perp}$ while $I_{\| \mid}$and $I_{\phi}$ remain unaffected [34]. By transforming the orbit averaged quasilinear operator to a stochastic differential equation and by using a modified Euler-Maruyama method to discretise it, the change in $I_{\perp}$ to apply is obtained in two steps as:

$$
\begin{aligned}
& \delta I_{\perp}=\xi \sqrt{2 \bar{D}_{R F}\left(I_{\perp}, I_{\|}, I_{\phi}\right) N_{A C C}}, \\
& \Delta I_{\perp}=\xi \sqrt{2 \bar{D}_{R F}\left(I_{\perp}+\delta I_{\perp}, I_{\|}, I_{\phi}\right) N_{A C C}}
\end{aligned}
$$

where $\xi$ is a random number uniformly distributed in the range $[-\sqrt{3}, \sqrt{3}]$, and

$$
\bar{D}_{R F}=\left|\frac{Z e}{2} \int_{\tau_{-}}^{\tau_{+}} v_{\perp}\left[E_{+} J_{n-1}\left(\frac{k_{\perp} v_{\perp}}{\omega_{c i}}\right)+E_{-} J_{n+1}\left(\frac{k_{\perp} v_{\perp}}{\omega_{c i}}\right)\right] \mathrm{e}^{i \nu(t)} d t\right|^{2},
$$

where $Z e$ is the ion charge and $\nu$ is the phase between the wave and the ion, as given by

$$
\frac{d \nu}{d t}=\omega-n \omega_{c i}-k_{\|} v_{\|}-k_{\perp} V_{D}
$$

The drift velocity $V_{D}$ is often neglected in the resonance condition but kept here for completeness. The time interval $\tau_{+}-\tau_{-}$should be centred around the stationary point $\frac{d \nu}{d t}=0$ and be long enough for the contribution to the integral from the vicinity of the stationary point to have converged (in most cases the integral can be evaluated by the stationary phase method).

The change in $\Delta I_{\perp}$ can be translated into local variables used in an orbit following code as: 


$$
\left\{\begin{aligned}
\Delta \mu & =\frac{n Z e}{m \omega} \Delta I_{\perp}, \\
\Delta W & =\Delta I_{\perp}, \\
\Delta v_{\|}^{2} & =\frac{2}{m}\left(1-\frac{n \omega_{c}}{\omega}\right) \Delta I_{\perp}, \\
\Delta \psi_{p} & =\frac{\frac{N}{\omega} \Delta W-\frac{m F}{B} \Delta v_{\|}}{\frac{Z e}{2 \pi}+\frac{m v_{\|}}{B} \frac{\partial F}{\partial \psi_{p}}}
\end{aligned}\right.
$$

where $m$ is the ion mass, $\psi_{p}$ is the poloidal flux coordinate, $F=R B_{\phi}$. The change of the flux surface location given above should be applied along an iso-B line passing through the resonance point (this is more easily achieved in orbit following codes that use the poloidal flux and an angle to determine position of the particle in real space whereas codes using cylindrical coordinates, $R, Z, \phi$ require an extra inversion; on the other hand the shift in the poloidal flux is normally small and often neglected).

In order to identify a resonance point RFOF calculates the frequency distance to the resonance $\delta \omega=\omega-n \omega_{c i}-k_{\|} v_{\|}-k_{\perp} V_{D}$ along the orbit, keeping in memory the two previous positions of the particle along the orbit. From the three points along the orbit, a second order polynomial $P_{\delta \omega}(t)$ is constructed. Its roots define the predicted time at the resonance. Polynomials are equally constructed from the $(R, Z)$ coordinates in the poloidal plane, along the orbit, $P_{R}(t)$ and $P_{Z}(t)$. From these polynomials and the predicted time at the resonance, RFOF predicts the position of the resonance. Conditions on the distance in frequency, time and position to the resonance are combined into a global criterion for assessing if an ion is in resonance with the wave. When a resonance is detected, the phase space portion of the Monte Carlo marker is updated according to Eq.(5). Fig.(4) illustrates the effect of the interaction with the ICRF waves for a $120 \mathrm{keV}$ fast ion orbit, with and without Doppler shift. The orbit is initially trapped. The green curve represents its shape before ICRH is applied. Then ICRF waves interact with the orbit at the locations represented by black points, leading to a deformation of the orbit to displace the banana tips towards the resonance region, given that the wave mainly gives kicks in the perpendicular direction of the particle. The final orbit is wider since in the case shown the fast ion gains velocity from its interaction with the waves.

Another important aspect of RFOF is that it adjusts the magnitude of the electric field to ensure that the correct ICRF power is absorbed by the resonating ions. This is necessary since it is not always possible to obtain 100\% consistency between codes solving the ICRF wave field (there are often slight variations in physics models between wave codes and the Fokker-Planck codes, which can result in somewhat different absorbed powers for the same wave field). Furthermore, when testing an orbit following Monte Carlo augmented by RFOF stand alone, an ad-hoc wave field profile is usually prescribed and its magnitude must be adjusted to the desired ICRF power, especially since the distribution function evolves and the absorption strength, therefore changes with time (the third harmonic absorption described in this paper is a prime example of this). For this reason, RFOF calculates the globally absorbed power with regular intervals and adjusts the wave field strength to maintain the requested absorbed ICRF power. Fig.(5) shows the positions where the deuterons receive kicks from the wave. The width of the resonance region is conditioned by the Doppler shift from Eq.(7). As can be seen, second harmonic $D$ heating also occurs at 

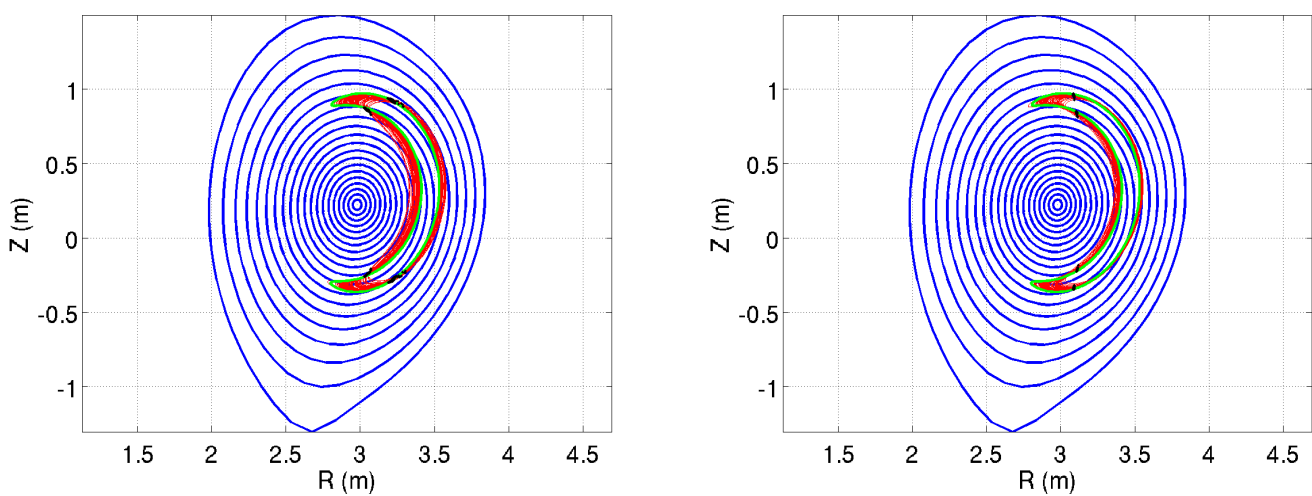

Figure 4: Example of a NBI 120 keV trapped ion orbit. Green orbit is with only NBI heating, while red orbit is with $N B I+I C R F$ heating; black points represent the location where the ion interacts with the ICRF waves. The left and right figures are with and without Doppler shift respectively.

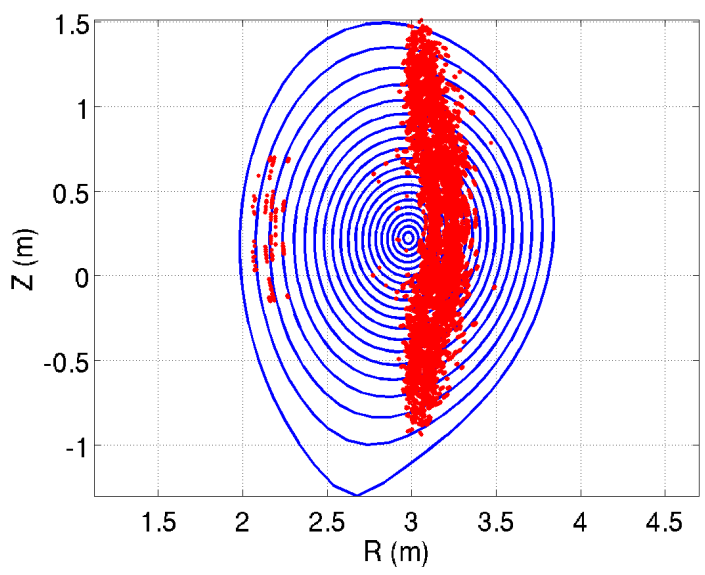

Figure 5: Location of RF-kicks to deuterons for JET discharge \#86459.

the edge on the high field side, but this absorption remains very low due to the low density an temperature of deuterons at the edge.

\section{Features of SPOT/RFOF simulation for JET shot \#86459}

Figure (6) shows the collisional power transferred from the non-thermal deuterons to the background plasma ions and electrons. The deuterium distribution function of the JET discharge \#86459 shown in Fig.(2) has been simulated with RFOF. As already mentioned the ICRF power and wave characteristics used in the simulation has been taken from a PION simulation [27], which obtained a good agreement between the measured and simulated total neutron rate of the discharge. According to the PION simulation, the power absorbed by the deuterons towards the end of the ICRF phase was $P_{D}=2 M W$, and other key parameters are given by $k_{\perp}=50 \mathrm{~m}^{-1}$ and $\left|E_{-} / E_{+}\right|=2.4$. The latter two were obtained for a toroidal mode number $N=30$, which corresponds to the peak of the mode number spectrum emitted by the ICRF antenna (the so called dipole phasing was used). Furthermore, the wave electric field was fixed with an ad-hoc profile such that the power density profile for the deuterons was similar to the one obtained in the PION simulation. As described above the magnitude of the electric field profile was adjusted in RFOF 

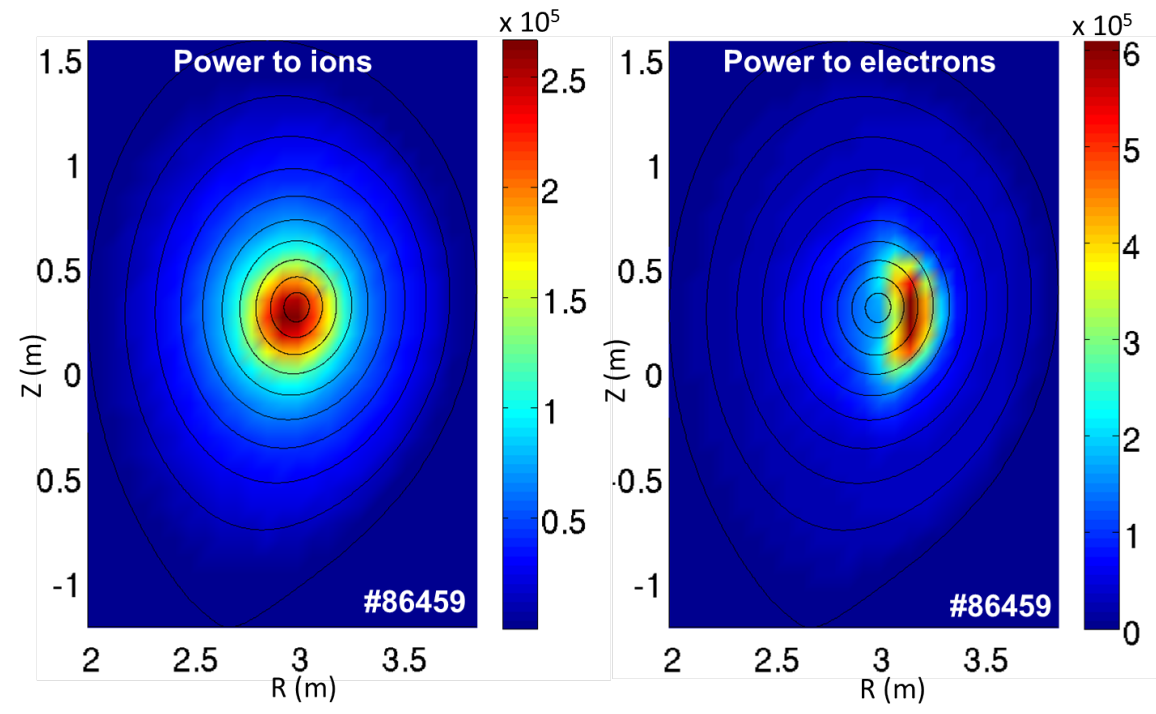

Figure 6: 2D profiles of power density to bulk ions (left figure) and electrons (right figure) for JET discharge \#86459, in $\mathrm{W} / \mathrm{m}^{3}$.

to maintain the absorbed power at 2MW. In the SPOT simulation, NBI injection was first applied to establish a NBI distribution of deuterons; the ICRF was subsequently switched on and the simulation was run up until a new steady state was reached. During the simulation all parameters were kept fixed except for the $E$-field normalisation.

As can be seen from Fig.(6), the power transfer to the ions is fairly evenly distributed poloidally, which is due to the fact that ion-ion collisions are strong only at low to moderate energies. Furthermore, the deuteron distribution is not strongly anisotropic in that energy range since low energy ions consist of dominant passing ions. On the other hand, the power to the electrons is peaked somewhat to the low field side of the magnetic axis. This is due to the fact that most of the high energy deuterons, that mainly collide with the electrons, are trapped with their turning points close to the resonance, in combination with the fact that trapped particles spend more time in the vicinity of the turning points than other locations along their orbits.

One can approximately insert the phase space position of the Monte Carlo integrator in an orbit classification diagram of the type described in [35], and one obtains the result displayed in Fig. (7). As expected this diagram shows that most of the deuterons above $200 \mathrm{keV}$ are trapped (region VII). Furthermore, their normalised toroidal angular momentum, $\hat{\psi}_{\phi}$, is typically below one, which indicates that they are in the potato regime (i.e. orbits that are not well described by normal trapped orbits). This emphasises the need for using models that accurately account for full orbit effects in simulations of this type of discharge.

The evolution of the square of the electric field normalisation factor $E_{\text {norm }}(t)$ is shown in Fig.(8). As can be seen, it decreases by roughly a factor of 5 as the energy of deuteron ions increases.

Since the absorbed power is proportional to the square of the electric field this means that the absorptivity is increased by a factor of 5 as the deuteron tail forms. This result is consistent with the findings of the PION simulation and emphasises the need for self-consistent simulations to analyse ICRF heating in the utilised scenario. 


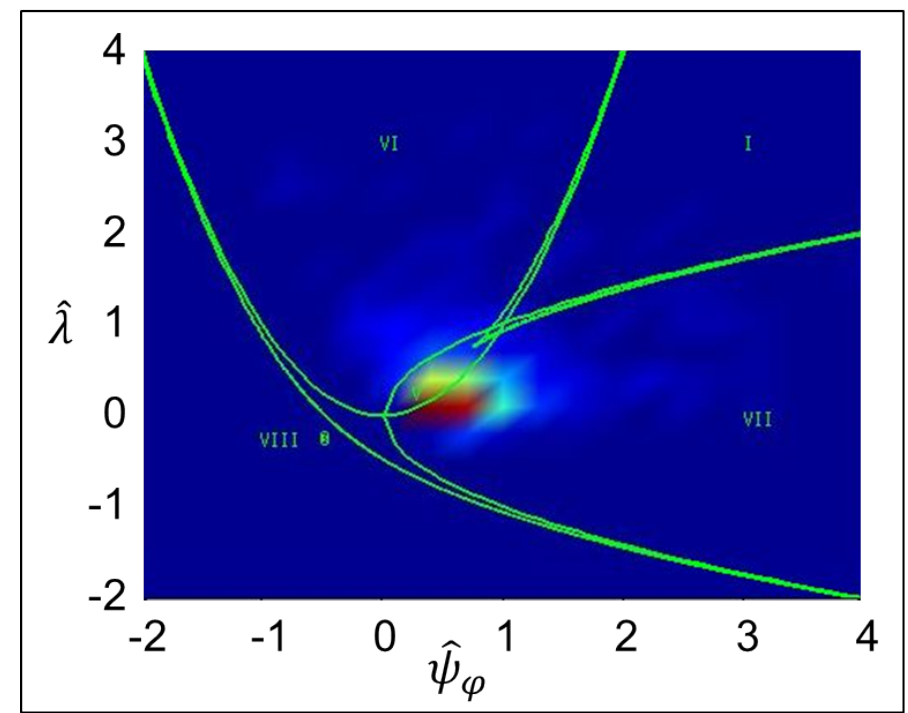

Figure 7: Orbit classification diagram.

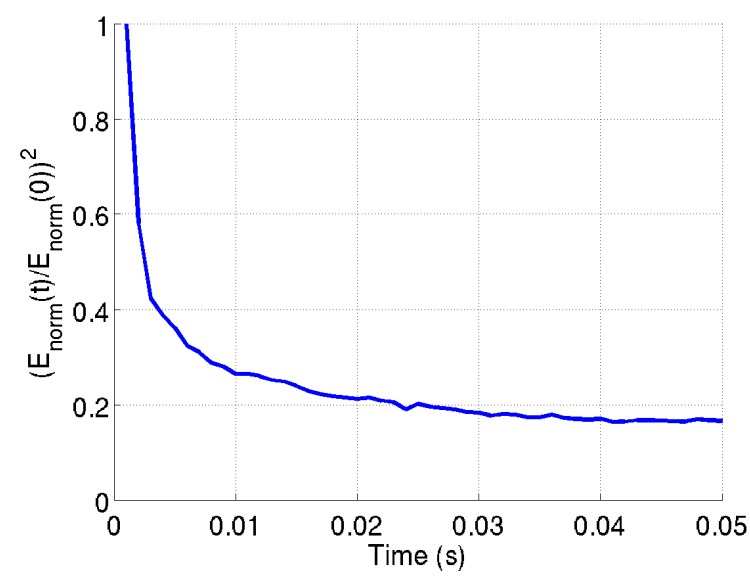

Figure 8: Time evolution of electric field normalisation factor. 


\section{Comparison with synthetic diagnostics}

A widely used technique for validating modelling codes against experiments is via the development of synthetic diagnostics for confronting the modelling with direct measured signals. This avoids the data deconvolution process that leads to uncertainties and loss of quantitative information from the diagnostics. Synthetic diagnostics consist of modelling the full response function of a detector and associated signal. The NBI+ICRH accelerated deuterium distribution function simulated by SPOT/RFOF and ASCOT/RFOF have been compared with neutron and gamma spectrometers for energy distribution, and with the neutron camera for spatial distribution of $D$ ions.

Simulated deuterium distribution functions are first compared with the signal from the TOFOR neutron spectrometer [36] via the calculation of the neutron emission spectrum using a Monte Carlo method as described in [37]. It allows a direct comparison between the predicted neutron energy spectrum and the measured data. The TOFOR is a time-of-flight spectrometer that measures the neutron energy by acquiring the time difference of detected neutrons between two sets of scintillators. Small times of flight correspond to high neutron energies, hence high reactant energies. The TOFOR is located in the JET roof laboratory and detects the plasma neutrons with a vertical line of sight directed towards the centre of the plasma with a radial sensitivity of $2.74<R<3.02$ $\mathrm{m}$. The neutron emission spectrum consists of neutrons from $D D$ reactions, including direct and multiple scattering processes between the two sets of scintillators, along with scattering of low energy ions in the far wall of the vacuum vessel. The former are taken into account in the diagnostic response function while the latter are treated as a separate spectral component. For NBI-heated plasmas, this description, neglecting the ion Larmor motion, is sufficient for reproducing TOFOR measured data, as shown in [37]. However, ICRH leads to an acceleration of fast ions in the perpendicular direction, inducing steeper spatial gradients of the fast ion distribution. In this case, Finite Larmor Radius effects have to be taken into account in the detector response function for correctly reconstructing the signal as measured by the spectrometer, see [38, 39] for details: in present experiments, the ICRH resonance layer is located at the outboard part of the TOFOR line of sight, meaning that ions travelling away from the detector, via their Larmor gyromotion, will not be detected. They correspond to down-shifted neutron energy, therefore neglecting FLR effects would lead to an overestimate of low energy neutrons. FLR effects are included in the TOFOR response function via the generation of random gyro-phases and the selection of ions that remain in the line of sight of the detector.

The initial comparison between the ASCOT/RFOF and SPOT/RFOF reconstructed signals and the TOFOR measurements is shown in Fig.(9). As can be seen, the overall match between both simulations and experiments is good. This comparison demonstrates the accurate description of the high energy tail and cut-off by the modelling: the TOFOR spectrum is in fact very sensitive to the ion high energy tail. The high energy cut-off is further discussed in section 7 .

Nevertheless, we also observe systematic discrepancies which are significant with respect to the uncertainty of the TOFOR measurements. The difference between the ASCOT and SPOT simulations is within the experimental uncertainties, so that the simulations cannot be told apart by the measurements in this discharge. Both simulations lie slightly below the TOFOR measurements for high neutron energies, corresponding to low times-of-flight (46ns-57ns), and slightly above for low energies, corresponding to high times-of-flight (62ns-69ns). Figure 10 illustrates the velocities of the fast ions generating the neutrons at 3,4 and $5 \mathrm{MeV}$ according to the SPOT simulation by showing the products of weight functions and the simulated central fast ion distribution function [40]. Most neutrons at low energies are generated due to ions with comparatively low energies. 

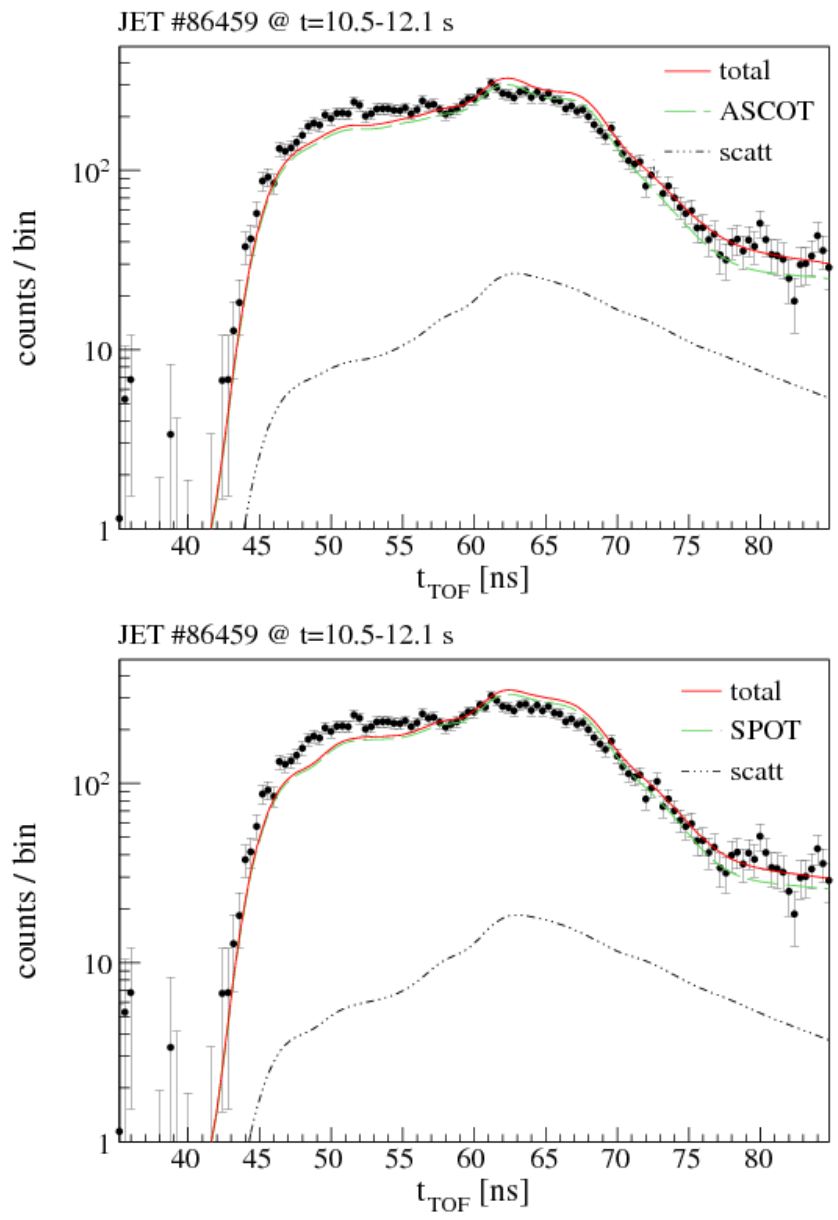

Figure 9: TOFOR neutron spectrum data (points) compared with reconstructed number of counts from ASCOT/RFOF (top figure) and SPOT/RFOF (bottom figure) deuterium distribution functions : black dashed dotted lines represent the scattered neutrons; dashed green lines represent the signal reconstructed from the modelling, and red solid line represents the sum of scattered and reconstructed data. 

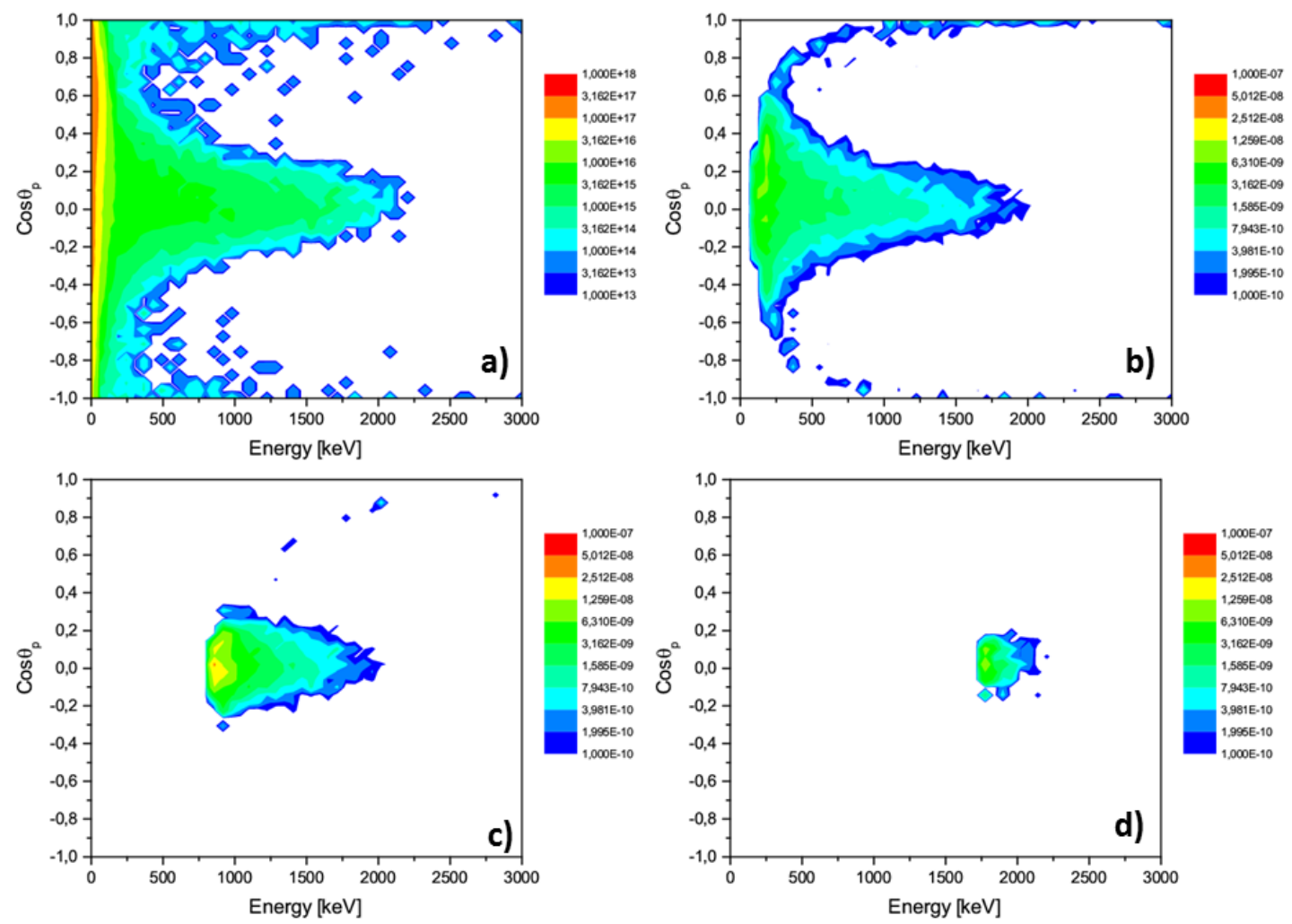

Figure 10: a) Total SPOT distribution function in the region $R=2.9$ to $3.1 \mathrm{~m}$ corresponding to the TOFOR line of sight; b) c) d) Velocity of fast ions generating neutrons at 3, 4 and $5 \mathrm{MeV}$ respectively, according to the product of weight functions with the SPOT simulated central fast ion distribution function.

An example is illustrated in figure 10-b showing neutrons with energies of $3 \mathrm{MeV}$ which have a nominal time-of-flight of about $60 \mathrm{~ns}$. For these and longer times-of-flight the simulations tend to show excess of neutrons (figure 9). Most neutrons at high energies are generated due to ions with comparatively high energies. Figure 10-c and 10-d illustrate the velocity space regions for $4 \mathrm{MeV}$ and $5 \mathrm{MeV}$ neutrons, corresponding to nominal times-of-flight of about $45 \mathrm{~ns}$ and $50 \mathrm{~ns}$, respectively. For these short times-of-flight the simulations show lack of neutrons (figure 9). Hence the SPOT simulation has a slight but significant lack of high-energy ions and surplus of lowenergy ions. Good qualitative matches with slight but significant quantitative discrepancies were also observed in benchmarking experiments of ASCOT simulations of NBI distributions against collective Thomson scattering measurements at ASDEX-Upgrade [41] and TEXTOR [42].

It is useful to mention that this comparison led to the identification of an issue with the initial SPOT/RFOF implementation, which was subsequently resolved. This shows the relevance of such a direct comparison and the strength of the TOFOR diagnostic for validating fast ion modelling.

Another comparison has been carried out between the simulated reconstructed signal and the measurements of the Single Crystal Diamond Detector (SDD) for neutron spectroscopy [43, 44]. The SDD line of sight has an angle of 47 degrees with respect to the plasma magnetic axis. The main interaction is the nuclear elastic scattering of the neutrons on ${ }^{12} C$ nuclei of the crystal, that mainly occurs at energies of NBI-heated plasmas. However when ICRH is applied, the inelastic scattering between neutrons and ${ }^{12} \mathrm{C}$ nuclei has to be included. Multiple scattering is also accounted 

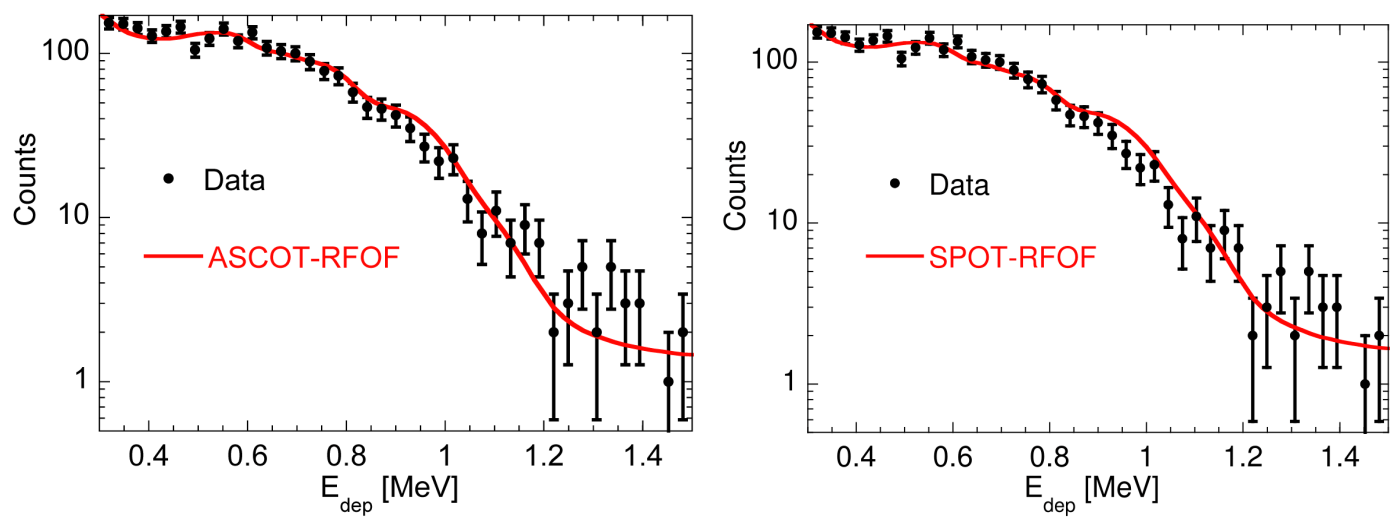

Figure 11: Diamond neutron spectrum data (points) compared with reconstructed number of counts from ASCOT/RFOF (left figure) and SPOT/RFOF (right figure) deuterium distribution functions (solid red lines).

for, but contributes to less than $1 \%$ of the signal. These processes create electron-hole pairs inside the crystal. The number of these pairs is related to the energy of the incident neutron, leading to ionization currents that can be detected and measured. The diamond detector response function is computed by the MCNP code [45] and the expected neutron spectrum is simulated by the GENESIS code [46].

The resulting reconstructed data using the ASCOT/RFOF and SPOT/RFOF distributions are compared with the SDD measurements in Fig.(11). As can be seen, there is an overall good agreement between the measured and reconstructed signals. Due to its semi-tangential line of sight, the SDD detector covers a different velocity space than TOFOR [40]. As for TOFOR, for 2D distribution functions consisting mostly of trapped ions, the spectra shown in Fig. (11) are mostly generated due to trapped ions. However, the diagnostic is more sensitive to the ions with negative pitch due to the geometry of the line-of-sight [40]. Hence, this comparison shows that the ion energy distribution is globally well described by the modelling. However, the very similar reconstructed data from ASCOT and SPOT tends to suggest that the SDD detector data are not fully sensitive to fine details of the ion distribution, while the neutron liquid scintillator exhibits more clear differences as described below, since in this discharge it provides data at higher statistics.

The NE213 organic liquid scintillator [47] is a neutron spectrometer with the same oblique line of sight of 47 degrees as the SDD detector, which makes a double pass through the plasma core. A neutron that scatters in the NE213 detector produces a scintillation light in a connected photomultiplier, resulting in a voltage allowing to record the full wave form of the pulse. The height of the pulse is related to the neutron energy deposited in the detector, allowing the reconstruction of the energy spectrum of the incident neutrons. The comparison between the experimental data and the synthetic reconstruction of the NE213 signal from ASCOT/RFOF and SPOT/RFOF distributions is shown in Fig.(12). An overall agreement is seen, except at high channels for the NE213/SPOT comparison, where SPOT slightly overestimates the number of high energy neutrons. Considering the good agreement with the TOFOR which has a vertical line of sight, this discrepancy tends to suggest an overestimate of the parallel velocities. This difference could be due to a too low statistic sample from the SPOT/RFOF simulation or a too loose quality of the Runge Kutta integration, but this requires further investigations.

Another comparison has been carried using a synthetic description of the High Purity Germanium (HpGe) gamma spectrometer. The HpGe spectrometer has a vertical line of sight and 

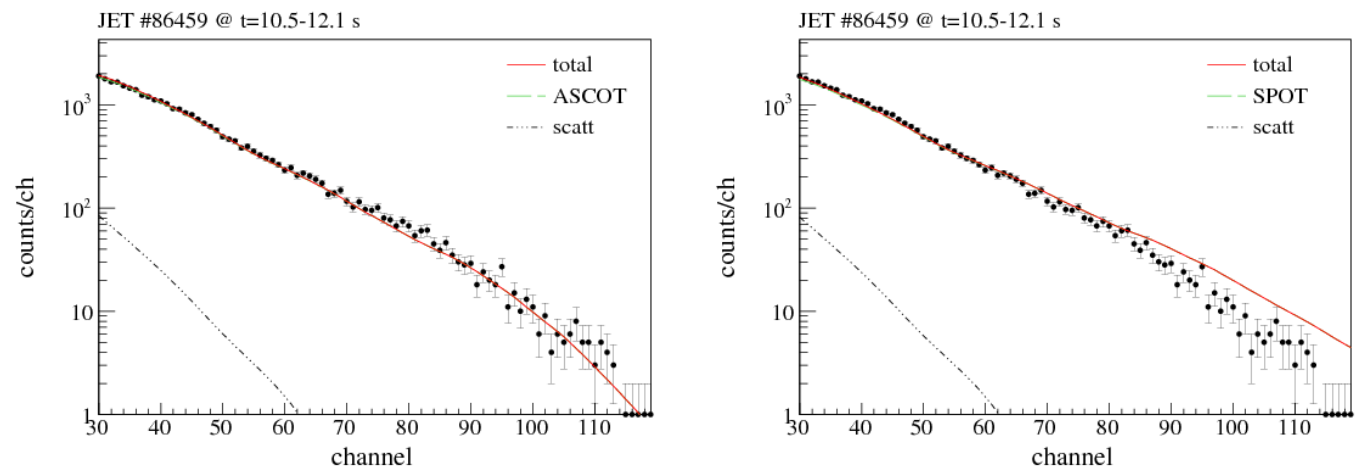

Figure 12: NE213 neutron spectrum data (points) compared with reconstructed number of counts from ASCOT/RFOF (left figure) and SPOT/RFOF (right figure) deuterium distribution functions: black dashed dotted lines represent the scattered neutrons; dashed green lines represent the signal reconstructed from the modelling, and red solid line represents the sum of scattered and reconstructed data.

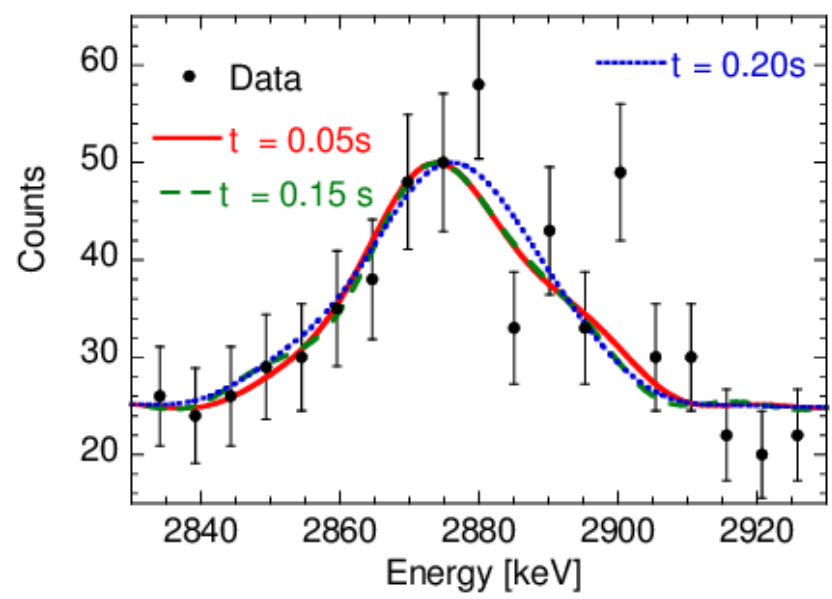

Figure 13: HpGe energy spectrum data (points) and reconstructed intensity from SPOT/RFOF distribution function (solid lines), for different time slices after the application of ICRH.

measures the Doppler broadening of gamma-ray emission profiles from multiple excited states, mainly from ${ }^{9} B e(D, n \gamma){ }^{10} B$ and ${ }^{9} B e(D, p \gamma){ }^{10} B e$ reactions. In this context, the Doppler broadening refers to the energy difference between gammas from reactants at rest and energetic reactants, hence providing some indication on the reactant energy (here the deuterium), see. e.g. [48] for details. Again, the GENESIS code has been used for simulating the gamma-ray emission profiles. As shown in [49], vertical gamma-ray spectroscopy is mostly sensitive to trapped ions at high Doppler shifts, making the HpGe spectrometer relevant for detecting the ICRH-accelerated fast deuteron tail. The comparison between the SPOT/RFOF reconstructed data and the gamma-ray intensity measurements versus the energy are shown in Fig.(13), for different time slices after the ICRH application. As can be seen, a very good agreement is observed, which is already there before the fast ion tail is completely established (the new steady-state being reached around 0.2 sec after switching on ICRH), since the high energy barrier or cut-off, as illustrated in Fig.(3), is early established in the NBI+ICRH heating phase.

Finally, the simulated distributions have been reconstructed for a comparison with the neutron camera, as shown in Fig.(14). The neutron camera consists of a set of scintillators assembled in a pair of collimator arrays, one vertical and one horizontal, allowing the reconstruction of the spatial 


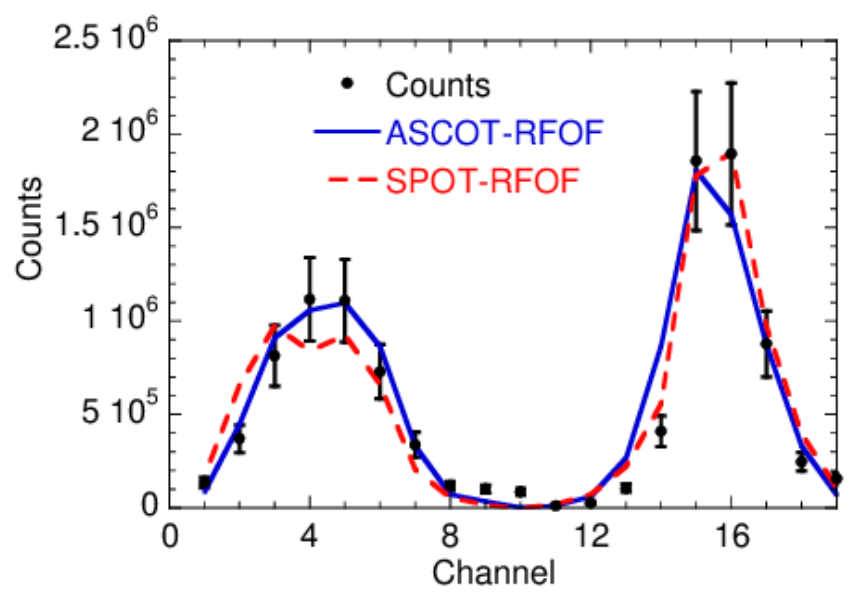

Figure 14: Neutron camera spatial data (black points) compared with reconstructed number of counts from ASCOT/RFOF (solid blue line) and SPOT/RFOF (dashed red line) deuterium distribution functions.

distribution of the emitted neutrons [50]. The first peak in the figure represents the horizontal camera while the second peak represents the vertical one. The results show an overall good agreement between the modelled and measured spatial distribution of emitted neutrons. ASCOT displays a slight discrepancy for the vertical camera while SPOT displays a slight discrepancy for the horizontal camera. The reason for these differences is unknown and needs further investigations.

\section{Experimental and simulated deuterium energy distribu- tion}

An alternative and complementary method to confront simulations with measurements is to compare the deuterium energy distribution. This comparison is more direct from the modelling point of view, but requires deconvolution processes from the diagnostics, leading to uncertainties regarding the global normalisation of the distribution functions. For this reason, diagnostics data have been adjusted to display the same ion distribution at $500 \mathrm{keV}$. Fig.(15) presents the comparison between TOFOR, BGO measurements and simulations from SPOT, ASCOT and PION codes. BGO is a bismuth germanate tangential gamma-ray scintillation spectrometer with a line of sight of $-0.2<Z<0.2 \mathrm{~m}$ [13].

As can be seen, TOFOR and BGO detectors exhibit a discrepancy for the energy cutoff. This difference may be due different velocity-space sensitivity, which would need further investigations by undertaking a weight function analysis as the one described in [51]. These differences could also be due the BGO horizontal line of sight, which misses the part of the ion distribution that includes banana tips, which could explain that perpendicular velocities tend to be underestimated. The discrepancy could also be due to the complexity of the gamma-ray deconvolution algorithm, based on the measurement of intensity peaks from different reactions involving deuterium; the most probable deuterium energy distribution is then estimated via a deconvolution using cross sections associated with each reactions [52]. Modelling from ASCOT, SPOT and PION show an overall good agreement with TOFOR, with a reasonable agreement for the energy cutoff, around $2.3 \mathrm{MeV}$. 


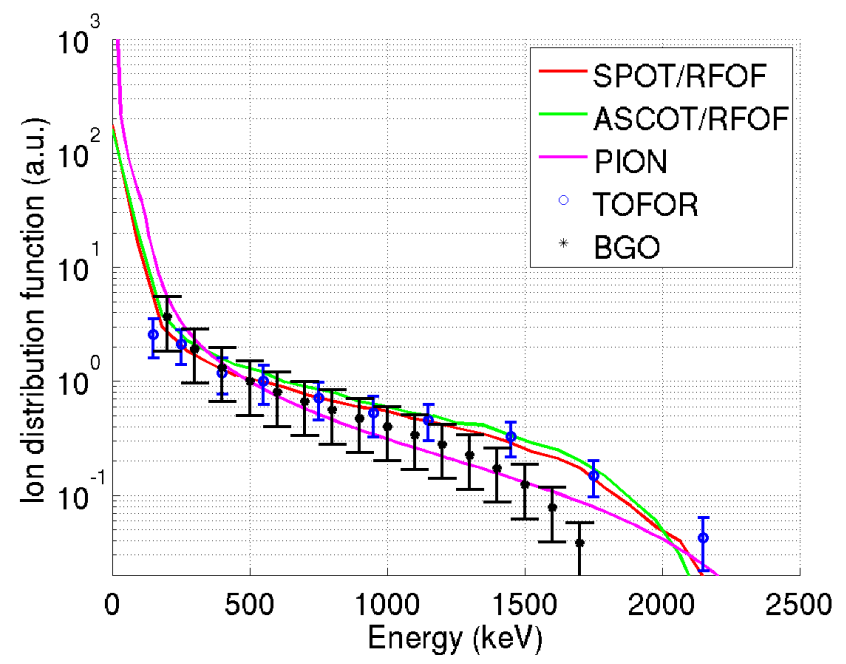

Figure 15: Comparison between the modelled deuterium energy distribution and experimental deconvoluted data, for discharge \#86459.

\section{Sawtooth control with NBI and ICRF heating}

Sawtooth activity is characterized by periodic crashes, i.e. sudden variations of the plasma density and temperature in the central region, where the safety factor $q$ is lower than 1 . A sawtooth crash is caused by the growth of an internal kink mode. The presence of a significant number of fast ions in the plasma centre can induce a stabilization of the sawteeth, leading to much longer periods between crashes. Understanding the sawtooth stabilization by fast ions is essential for controlling them. In ITER, monster sawteeth induced by fast ion stabilization may trigger neoclassical tearing modes (NTMs) leading to a significant degradation of confinement [53]. Besides, small period sawteeth may be desirable for expelling helium ashes and allowing deuterium and tritium entering the plasma core (i.e. crossing the $q=1$ surface).

Four discharges of the JET 2014 fusion product studies experiments have been analysed for they exhibit a significant sawtooth activity, with a maximum sawtooth period of about 2.5 seconds. The study reported here, fully detailed in [54], aims at demonstrating the stabilization effect of third harmonic NBI+ICRH heated deuterium ions within the Porcelli model [55]. This model assumes that a sawtooth remains stable as long as the potential energy functional $\delta \hat{W}$ is positive:

$$
\delta \hat{W}=\delta \hat{W}_{M H D}+\delta \hat{W}_{k i n}=\delta \hat{W}_{M H D}+\delta \hat{W}_{N B I}+\delta \hat{W}_{I C R H},
$$

where $\delta \hat{W}_{M H D}$ is the MHD potential energy functional, usually negative (destabilizing), here calculated from the internal kink mode growth rate simulated from the MISHKA code [56]; $\delta \hat{W}_{k i n}$ is the kinetic potential energy functional, usually positive (stabilizing), here consisting of contributions from NBI and ICRH heating, respectively $\delta \hat{W}_{N B I}$ and $\delta \hat{W}_{I C R H}$. The former is calculated using an analytical expression while the latter is computed using the HAGIS code [57]. Both used ion distributions and pressure profiles from SPOT, the NBI ion distribution being computed by NEMO/SPOT and the NBI+ICRH accelerated ion distribution being computed by SPOT/RFOF.

Results indicate that, taking into account only the bulk plasma equilibrium and the fast particle distribution, the potential energy functional increases with time, in contradiction with the observed sawtooth crashes. However, tornado modes are observed in two of the studied discharges. Tornado 
modes are known to expel fast particles from the core plasma inside the $q=1$ surface, thus leading to a broadening of the fast ion distribution function, see e.g. [58]. When this broadening is taken into account in the Porcelli model, the potential energy function actually decreases until it becomes negative, leading to sawtooth crashes as observed in the experiment. In the other two discharges, no tornado mode is observed and the neutron cameras confirm that the fast ion distribution is not broadened. The potential energy functional for these discharges is found to be increasing, i.e. fast ions are still stabilizing. In particular, it is very far from the threshold in the case of the discharge exhibiting the longest sawtooth (about $2.5 \mathrm{~s}$ ), due to a large energy content. Yet sawtooth crashes occur; they could be due to ELM bursts, observed just before the crashes, which trigger an inward propagating cold front reaching the $q=1$ surface.

\section{Conclusion}

Results of recent JET experiments aimed at studying fusion products have here been used for validating ICRF modelling codes used within the EU-IM modelling framework. The analysed experiments employed third harmonic ICRF acceleration of neutral beam injected deuterons, which resulted in a spectacular enhancement of the $D D$ fusion reaction rate due to the applied ICRF power. The TOFOR time-of-flight spectrometer provided crucial information on the distribution function of the reacting species, which was complemented by data from diamond and liquid scintillator neutron detectors, gamma-ray HpGe spectrometers, and neutron cameras. The measurements with these instruments have been compared to simulation results obtained with the orbit following Monte Carlo codes ASCOT and SPOT augmented with the recently developed RFOF library. The latter updates the Monte Carlo markers in orbit following codes to account for resonant ICRF wave-particle interaction.

The scenario with combined $D \mathrm{NBI}$ and $\omega=3 \omega_{c D}$ ICRF heating is a challenging scenario to model, and therefore provides a rather stringent test of the modelling codes. It also has the advantage of not requiring experimental quantities that are difficult to obtain with accuracy from the experiments (e.g. like the concentration of the resonating species during minority heating) as input to the simulations. In terms of the requirements on the modelling tools, it has been demonstrated that finite orbit width effects (including orbits in the potato regime) must be taken into account for detailed comparisons with the diagnostics used. Furthermore, it is crucial to have a capability to perform self-consistent simulations whereby information on the evolution of the deuteron distribution function is used to update the dielectric properties in the wave deposition code. For example, in the simulated scenario it was found that the absorptivity by the resonating deuterons was enhanced by a factor 5-10 as compared to an NBI only phase when the distribution function evolved towards a steady state in the presence of ICRF. Because the orbit following Monte Carlo codes used in this study have not yet been interfaced self-consistently to wave deposition codes, the wave characteristics used in the simulations had to be taken from runs of the PION code (which performs internally self-consistent calculations). The results of the latter were able to reproduce the measured neutron rates well, and its results are broadly in line with the findings of the Monte Carlo codes, giving confidence in the overall consistency of the simulations.

The deuteron distribution functions simulated with the ASCOT/RFOF and SPOT/RFOF packages were used as input to dedicated synthetic diagnostics for the neutron time-of-flight TOFOR spectrometer, and also used to model the response of neutron diamond, neutron liquid, gamma-ray HpGe spectrometers, and neutron cameras. A good agreement was found between the 
modelling and neutron and gamma-ray spectrometers. Furthermore, a good consistency between modelling and the neutron cameras was found, indicating that the 2D spatial distribution of the fast $D$ ions was well simulated. The comparison between the different modelling tools and deconvoluted data from neutron and gamma BGO spectrometers indicate that the "barrier region" where the wave-particle interaction is weak is well predicted by the modelling tools. These results increase the confidence in the modelling tools, and indicate that no major physics effect is unaccounted for.

The results presented in this paper have demonstrated the value of making detailed comparisons with diagnostics and cross benchmarking of codes. The exercise has initiated further investigations to identify the reason for slight differences in the results between the Monte Carlo codes. In addition, these comparisons serve as an important contribution to the ongoing validation process of ICRF modelling codes within the EU-IM framework.

Finally, a sawtooth activity has been observed in these experiments, and interpreted using SPOT/RFOF simulations in the framework of the Porcelli's theoretical model. In particular, NBI+ICRH accelerated ions are found to have a strong stabilizing effect. However, sawtooth crashes still occur, especially due to tornado modes induced by fast ions [54]. This sawtooth stabilization by fast ions is a major issue for ITER, which should have significant population of fusion-born alpha particles. The eventual crashes of fast ion stabilised sawteeth have a tendency to create seed island capable of triggering Neoclassical Tearing modes, which can degrade the performance of a fusion plasma.

\section{Acknowledgments}

This work has been carried out within the framework of the EUROfusion Consortium and has received funding from the Euratom research and training programme 2014-2018 under grant agreement No 633053. The views and opinions expressed herein do not necessarily reflect those of the European Commission.

\section{References}

[1] The JET Team (presented by M Keilhacker) 1997 Plasma Phys. Control. Fusion 39 B1.

[2] P. Thomas et al., Phys. Rev. Lett. V.80 (1998) 5548

[3] S.E. Sharapov et al., Fusion Science and Technology, v.53 (2008) 989

[4] J.D. Strachan et al 1997 Plasma Phys. Control. Fusion 39 B103

[5] B.C. Stratton et al, Nucl. Fusion 391309 (1999)

[6] M. Mantsinen et al, Phys. Rev. Letters, 88 (2002) 10

[7] M. Nocente et al, Nucl. Fusion 52 (2012) 063009

[8] National Physical Laboratory, Kaye \& Laby. Tables of Physical \& Chemical Constant, Chapter 4, Section 4.7, Subsect. 4.7.4 Nuclear Fusion. (2014).

[9] L.-G. Eriksson et al, Nucl. Fusion 33 (1993) 1037.

[10] L.-G. Eriksson et al, Nucl. Fusion, Vol. 38, No. 2 (1998)

[11] T. Hellsten et al, "RF Heating for Fusion Product Studies", 21st Topical Conference on RF Power in Plasmas, Lake Arrowhead, California (2015)

[12] M. Gatu Johnson et al, Nuc. Instr. Meth. A 591417 (2008) 
[13] V. G. Kiptily, et al, Nuclear Fusion 42, 999 (2002)

[14] M. Tardocchi et al, Plasma Phys. Control. Fusion 55074014 (2013)

[15] T. Hellsten et al, Nucl. Fusion 44892 (2004)

[16] L.-G. Eriksson et al, Nucl. Fusion 39337 (1999)

[17] G. Falchetto et al, Nucl. Fusion 54043018 (2014)

[18] P. U. Lamalle, PhD thesis - Université de Mons (1994) LPP-ERM/KMS Laboratory Report 101

[19] R. Dumont et al, Nuclear Fusion 53, 013002 (2013)

[20] L. Villard, et al., Nuclear Fusion 35, 1173 (1995)

[21] M. Brambilla, Plasma Phys. Control. Fusion 411 (1999)

[22] T. Johnson et al, AIP Proc. 1406, 373 (2011)

[23] M. Schneider et al, Plasma Phys. Control. Fusion 47 (2005) 2087-2106

[24] E. Hirvijoki et al. Computer Physics Communications 185 (2014) 1310-1321

[25] M. Schneider et al, 14th ITPA on Energetic Particle Physics, ITER HQ, France (2015)

[26] R. Dumont et al, "Advanced simulation of energetic ion populations in the presence of NBI and RF sources", EPS conference, Berlin (2015)

[27] M. Mantsinen et al, "Analysis of ICRF heating and ICRF-driven fast ions in recent JET experiments", IAEA Technical Meeting on Energetic Particles, Vienna (2015)

[28] M. Schneider et al, Nuclear Fusion 51 (2011) 063019

[29] O. Asunta et al, Comp. Phys. Comm., 188 (2015) 33-46

[30] T.H Stix "waves in Plasmas" American Institute of Physics 1992, ISBN 0-88318-859-7; A. Bécoulet, D.J. Gambier, A. Samain, Phys. Fluids B 3 (1991) 137; P. Helander and M. Lisak, Physics of Fluids B, Volume 4 (1992) 1927.

[31] M. Mantsinen at al. Nuclear Fusion 39 (1999) 459.

[32] A. Salmi et al, Plasma Phys. Control. Fusion 48 (2006) 717-726

[33] M. Schneider et al, "Benchmarking Neutral Beam Injection codes within the European Integrated Modelling framework", Proc. EPS Lisbon (2015)

[34] L.-G. Eriksson, M. Schneider, Physics of Plasmas, 12, 072524 (2005)

[35] L.-G. Eriksson and F. Porcelli, sma Phys. Control. Fusion (2001) 145-182

[36] Gatu Johnson M et al, Nucl. Instrum. Methods A, 591417 (2008)

[37] C Hellesen et al, Plasma Phys. Control. Fusion 52 (2010) 085013

[38] J. Eriksson et al, Plasma Phys. Control. Fusion 55015008 (2013)

[39] C. Hellesen et al, Nucl. Fusion 53 (2013) 113009

[40] A.S. Jacobsen et al, Nucl. Fusion 55 (2015) 053013

[41] M. Salewski et al. Nucl. Fusion 50 (2010) 035012

[42] D. Moseev et al, Plasma Phys. Control. Fusion 53105004 (2011)

[43] M. Nocente et al. Rev. Sci. Instrum. 86 (2015) 103501

[44] C. Cazzaniga et al. Rev. Sci. Instrum. 85 (2014) 043506

[45] General Monte Carlo N-Particle (MCNP) Transport Code, https://mcnp.lanl.gov/

[46] M. Tardocchi et al, Phys. Rev. Lett. 107, 205002 (2011)

[47] F. Binda et al, Review of Scientific Instruments, 85 11E123 (2014) 
[48] Nocente, M. "Neutron and Gamma-Ray Emission Spectroscopy as Fast Ion Diagnostics in Fusion Plasmas" PhD thesis, https://boa.unimib.it/handle/10281/28397 (2012)

[49] M. Salewski et al, Nucl. Fusion 55 (2015) 093029

[50] M. Riva et al, Fus. Eng. and Design 86 (2011) 1191.

[51] J. Eriksson et al, Nucl. Fusion 55 (2015) 123026

[52] A.E. Shevelev et al. Nucl. Fusion 53 (2013) 123004

[53] I.T. Chapman et al, Nucl. Fusion 53 (2013) 066001

[54] J.-B. Girardo et al, accepted to Physics of Plasmas (2016)

[55] F. Porcelli, Plasma Phys. Control. Fusion 331601 (1991)

[56] A.B. Mikhailovskii et al, Plasma Physics Reports 23844 (1997)

[57] S.D. Pinches et al, Comp. Phys. Comm. 111, 133 (1998)

[58] T. Gassner, et al, Physics of Plasmas 19, 032115 (2012) 